\title{
POSITION VECTORS OF NUMERICAL SEMIGROUPS
}

\author{
LANCE BRYANT AND JAMES HAMBLIN \\ Shippensburg University \\ 1871 Old Main Dr. \\ Shippensburg, PA 17257
}

\begin{abstract}
We provide a new way to represent numerical semigroups by showing that the position of every Apéry set of a numerical semigroup $S$ in the enumeration of the elements of $S$ is unique, and that $S$ can be re-constructed from this "position vector." We extend the discussion to more general objects called numerical sets, and show that there is a one-to-one correspondence between $m$-tuples of positive integers and the position vectors of numerical sets closed under addition by $m+1$. We consider the problem of determining which position vectors correspond to numerical semigroups.
\end{abstract}

\section{INTRODUCTION}

We let $\mathbb{N}$ and $\mathbb{N}_{0}$ denote the positive and nonnegative integers, respectively. A numerical semigroup $S$ is a subsemigroup of $\mathbb{N}_{0}$ that contains 0 and has finite complement in $\mathbb{N}_{0}$. For two elements $u$ and $u^{\prime}$ in $S, u \preceq_{S} u^{\prime}$ if there exists an $s \in S$ such that $u+s=u^{\prime}$. This defines a partial ordering on $S$. The minimal elements in $S \backslash\{0\}$ with respect to this ordering form a unique minimal set of generators for $S$, which is denoted by $\left\{a_{1}, a_{2}, \ldots, a_{\nu}\right\}$ where $a_{1}<a_{2}<\cdots<a_{\nu}$. The semigroup $S=\left\{\sum_{i=1}^{\nu} c_{i} a_{i}: c_{i} \geq 0\right\}$ is represented using the notation $S=\left\langle a_{1}, \ldots, a_{\nu}\right\rangle$. Since the minimal generators of $S$ are distinct modulo $a_{1}$, the set of minimal generators is finite. Furthermore, having finite complement in $\mathbb{N}_{0}$ is equivalent to $\operatorname{gcd}\left\{a_{i}: 1 \leq i \leq \nu\right\}=1$.

The number of minimal generators of a semigroup $S$ is called the embedding dimension of $S$, and is denoted by $\nu=\nu(S)$. The element $a_{1}$ is called the multiplicity of $S$, and is also denoted by $e_{0}(S)$. When $S \neq \mathbb{N}_{0}$, we always have $2 \leq \nu(S) \leq e_{0}(S)$.

For $0 \neq n \in S$, the Apéry set of $S$ with respect to $n$ is the set

$$
\operatorname{Ap}(S, n)=\{w \in S: w-n \notin S\} .
$$

Every numerical semigroup containing $n$ has a unique Apéry set with respect to $n$ from which much can be gleaned. Indeed, in [3] the Apéry set is described as the most versatile tool in numerical semigroup theory.

E-mail address: lebryant@ship.edu, jehamb@ship.edu. 
We take the representation by an Apéry set a step further. Consider the semigroup $S=\langle 4,7,9\rangle$. We have $\operatorname{Ap}(S, 4)=\{0,7,9,14\}$. If we enumerate the elements of $S$ so that $S=\left\{\lambda_{0}, \lambda_{1}, \lambda_{2}, \ldots\right\}$, where $\lambda_{i}<\lambda_{j}$ whenever $i<j$, then $\operatorname{Ap}(S, 4)=\left\{\lambda_{0}, \lambda_{2}, \lambda_{4}, \lambda_{8}\right\}$. We can say that the position of the Apéry set in the enumeration is given by $(0,2,4,8)$. It will be convenient to remove 0 from this vector and consider the difference of the components. For example, we represent $S=\langle 4,7,9\rangle$ (as a semigroup containing 4 ) with the vector $(2,2,4)$ instead of $(0,2,4,8)$. This new vector has nonnegative integer components and stores equivalent information about the semigroup. We make the following definition.

Definition 1.1. Let $S=\left\{\lambda_{0}, \lambda_{1}, \lambda_{2}, \ldots\right\}$ be a numerical semigroup containing $n \neq 0$ such that $\lambda_{i}<\lambda_{j}$ whenever $i<j$. If $\operatorname{Ap}(S, n)=\left\{\lambda_{0}, \lambda_{x_{1}}, \lambda_{x_{2}}, \ldots, \lambda_{x_{n-1}}\right\}$, then the $(n-1)$-tuple $\left(x_{1}, x_{2}-x_{1}, x_{3}-x_{2}, \ldots, x_{n-1}-x_{n-2}\right)$ is called the position vector of $S$ with respect to $n$, and is denoted by $\operatorname{pv}_{n}(S)$.

We show in Corollary 2.11 that if $S$ and $T$ are semigroups containing $n \neq 0$, then $S=T$ if and only if $\operatorname{pv}_{n}(S)=\mathrm{pv}_{n}(T)$. Thus, a position vector is a representation of the semigroup. This is a rather remarkable fact. Consider the semigroup $\langle 4,7,9\rangle$. Since the position vector is $(2,2,4)$ (which means that the elements of $\operatorname{Ap}(\langle 4,7,9\rangle, 4)$ are in positions $0,2,4$, and 8 in the enumeration), no other semigroup can have an Apéry set with elements in those same positions. This is certainly not true for minimal generating sets: the position of the minimal generators of $\langle 4,7,9\rangle$ and $\langle 4,6,9\rangle$ are the same, namely the first, second, and fourth elements in the enumeration. Nonetheless, the position vectors are $(2,2,4)$ and $(2,2,5)$ respectively.

Not every vector of positive integers is the position vector of a numerical semigroup. In Section 2 we extend our consideration to more general objects than numerical semigroups, which we call numerical sets, and show that there is a one-to-one correspondence between elements of $\mathbb{N}^{n-1}$ and the position vectors of numerical sets closed under addition by $n$. Although we do not stress the fact in this paper, a numerical set $I$ can always be interpreted as a relative ideal of a semigroup contained in $I$, and so, in a sense, we have not extended beyond the theory of numerical semigroups.

Among the position vectors of numerical sets, we examine the problem of determining which represent numerical semigroups in Section 3 ,

\section{Position Vectors of numerical Sets}

As stated in the introduction, we need to work with objects more general than numerical semigroups.

Definition 2.1. A numerical set $I$ is a subset of $\mathbb{N}_{0}$ that contains 0 and has finite complement in $\mathbb{N}_{0}$.

Remark 2.2. A numerical set $I$ is closed under addition if and only if it is a numerical semigroup. When $I$ is not closed under addition, it is a relative ideal of the numerical semigroup $I-I$. Thus, we can think of the numerical sets as a certain collection of relative ideals which includes numerical semigroups. See [1] for information on relative ideals and [5, 6] for numerical sets. 
We need to define the position vector of a numerical set as we did for numerical semigroups in the introduction. We begin with a preliminary definition.

Definition 2.3. For $n \neq 0$, let $\Gamma_{n}$ be the collection of numerical sets $I$ such that $n+i \in I$ for all $i \in I$.

For $I \in \Gamma_{n}$, we can define the Apéry set with respect to $n$ as

$$
\operatorname{Ap}(I, n)=\{w \in I: w-n \notin I\} .
$$

As with numerical semigroups, it is not difficult to see that $\operatorname{Ap}(I, n)$ contains exactly $n$ elements of $I$ including 0 . Now we can define the position vector of the numerical set.

Definition 2.4. Let $I=\left\{\lambda_{0}, \lambda_{1}, \lambda_{2}, \ldots\right\}$ be in $\Gamma_{n}$ such that $\lambda_{i}<\lambda_{j}$ whenever $i<j$. If $\operatorname{Ap}(I, n)=\left\{\lambda_{0}, \lambda_{x_{1}}, \lambda_{x_{2}}, \ldots, \lambda_{x_{n-1}}\right\}$, the $(n-1)$-tuple $\left(x_{1}, x_{2}-x_{1}, x_{3}-x_{2}, \ldots, x_{n-1}-x_{n-2}\right)$ is called the position vector of $I$ with respect to $n$, and is denoted by $\mathrm{pv}_{n}(I)$.

A numerical set has multiple position vectors, but no two can have the same length. Therefore, $f: \Gamma_{n} \mapsto \mathbb{N}^{n-1}$ such that $f(I)=\operatorname{pv}_{n}(I)$ is a well-defined function. The goal of this section is to prove that $f$ is a one-to-one correspondence, which is proven in Theorem 2.10 .

We first establish Proposition 2.6. which contains a result about permutations on the set $1,2, \ldots, m$.

Definition 2.5. Let $\pi=\left[\pi_{1} \cdots \pi_{m}\right]$ be a permutation of the set $\{1, \ldots, m\}$. Then the conversion vector of $\pi$ is $r=\left(r_{1}, \ldots, r_{m}\right)$, where $r_{i}=\mid\left\{j: j<i\right.$ and $\left.\pi_{j}<\pi_{i}\right\} \mid$.

Notice that in Definition 2.5, we have $0 \leq r_{i} \leq i-1$ for all $1 \leq i \leq m$. Moreover, Proposition 2.6 reveals that every vector $\left(r_{1}, \ldots, r_{m}\right)$ with this restriction is the conversion vector of a unique permutation. This result is similar to a well-known result about inversion vectors of permutations, see [7, 8].

Proposition 2.6. For a fixed integer $m \geq 1$, let $r=\left(r_{1}, r_{2}, \ldots, r_{m}\right)$ be a vector such that $0 \leq r_{i} \leq i-1$, for $1 \leq i \leq m$. Then there is a unique permutation $\pi$ of the set $\{1, \ldots, m\}$ for which $r$ is its conversion vector.

Proof. Since there are exactly $m$ ! such vectors and $m$ ! permutation of length $m$, it suffices to show that each vector is the conversion vector of some permutation. The uniqueness follows by counting.

We will proceed by induction on $m$. If $m=1$, then $r=(0)$ and $\pi=[1]$ has $r$ as its conversion vector. Now assume that $m \geq 2$ and that there is a permutation $\sigma=$ $\left[\sigma_{1} \sigma_{2} \cdots \sigma_{m-1}\right]$ with the conversion vector $\left(r_{1}, r_{2}, \ldots, r_{m-1}\right)$. For each $1 \leq i \leq m-1$, define

$$
\delta_{i}= \begin{cases}1 & \text { if } \sigma_{i}>r_{m} \\ 0 & \text { otherwise }\end{cases}
$$

Let $\pi=\left[\left(\sigma_{1}+\delta_{1}\right)\left(\sigma_{2}+\delta_{2}\right) \cdots\left(\sigma_{m-1}+\delta_{m-1}\right)\left(r_{m}+1\right)\right]$. It is not difficult to see that $\pi$ is a permutation, and that $\left(\sigma_{i}+\delta_{i}\right)<\left(\sigma_{j}+\delta_{j}\right)$ if and only if $\sigma_{i}<\sigma_{j}$, for $1 \leq i \leq m-1$. Thus the conversion vector of $\pi$ is $r$. 
Example 2.7. Consider the permutation [42351]. We can directly observe that the conversion vector is $(0,0,1,3,0)$. Conversely, since the proof in Proposition 2.6] is constructive, we can recursively recover the permutation as follows:

$$
\begin{aligned}
{[(0+1)] } & =[1] \\
{[(1+1)(0+1)] } & =[21] \\
{[(2+1)(1+0)(1+1)] } & =[312] \\
{[(3+0)(1+0)(2+0)(3+1)] } & =[3124] \\
{[(3+1)(1+1)(2+1)(4+1)(0+1)] } & =[42351] .
\end{aligned}
$$

If the elements of the Apéry set of a numerical set $I$ are known, the position vector can be determined without considering the enumeration of all the elements of $I$. To see this, let $\operatorname{Ap}(I, n)=\left\{\lambda_{x_{0}}, \lambda_{x_{1}}, \ldots, \lambda_{x_{n-1}}\right\}$. We write $\lambda_{x_{i}}=n k_{i}+\pi_{i}$, where $0 \leq \pi_{i}<n$. Notice that the elements of $\operatorname{Ap}(I, n)$ form a complete residue system modulo $n$ and $\pi_{0}=0$. Thus, $\pi=\left[\pi_{1} \cdots \pi_{n-1}\right]$ is a permutation of the set $\{1, \ldots, n-1\}$ with a corresponding conversion vector $r=\left(r_{1}, r_{2}, \ldots, r_{n-1}\right)$.

Theorem 2.8. Let $I \in \Gamma_{n}$ and $\operatorname{Ap}(I, n)=\left\{w_{0}, w_{1}, \ldots, w_{n-1}\right\}$, where $w_{i}<w_{j}$ whenever $i<j$. We write $w_{i}=n k_{i}+\pi_{i}$, with $0 \leq \pi_{i}<n$. Let $r=\left(r_{1}, \ldots, r_{n-1}\right)$ be the conversion vector of $\pi=\left[\pi_{1} \pi_{2} \cdots \pi_{n-1}\right]$. Then $\operatorname{pv}_{n}(I)=\left(v_{1}, v_{2}, \ldots, v_{n-1}\right)$, where $v_{1}=k_{1}+1$ and $v_{i}=i\left(k_{i}-k_{i-1}\right)+\left(r_{i}-r_{i-1}\right)$, for $2 \leq i \leq n-1$.

Proof. Let $I=\left\{\lambda_{0}, \lambda_{1}, \ldots\right\}$ and $w_{i}=\lambda_{x_{i}}$. Since $0=w_{0}=\lambda_{x_{0}}$, we have $x_{0}=0$. Next we show that $x_{i}=i k_{i}-\sum_{j=0}^{i-1} k_{j}+r_{i}+1$, for $1 \leq i \leq n-1$. To do this, we compute the number of elements in $I$ that are strictly less than $n k_{i}$ for $1 \leq i \leq n-1$. The sequence $\left(k_{0}, k_{1}, \ldots, k_{n-1}\right)$ is non-decreasing, and we set $l$ to be the largest index such that $k_{i}=k_{l}$. Now $s \in I$ and $n k_{l} \leq s \leq n k_{l}+(n-1)$ if and only if $s=\lambda_{x_{j}}+n\left(k_{l}-k_{j}\right)$ for some $0 \leq j \leq l$. Thus

$$
\begin{aligned}
\left|\left\{0,1,2, \ldots, n k_{i}-1\right\} \cap I\right| & =\left|\left\{0,1,2, \ldots, n k_{l}-1\right\} \cap I\right| \\
& =\sum_{j=0}^{l}\left(k_{l}-k_{j}\right) \\
& =\sum_{j=0}^{i-1}\left(k_{i}-k_{j}\right) \\
& =i k_{i}-\sum_{j=0}^{i-1} k_{j} .
\end{aligned}
$$


Next, $s \in I$ and $n k_{i} \leq s<\lambda_{x_{i}}$ if and only if $s=\lambda_{x_{j}}+n\left(k_{i}-k_{j}\right)=n k_{i}+\pi_{j}$, for some $0 \leq j<i$ and $\pi_{j}<\pi_{i}$. There are $r_{i}+1$ such elements. Therefore, we have

$$
\begin{aligned}
x_{i} & =\left|\left\{\lambda_{0}, \lambda_{1}, \ldots, \lambda_{x_{i}-1}\right\}\right| \\
& =i k_{i}-\sum_{j=0}^{i-1} k_{j}+r_{i}+1
\end{aligned}
$$

Recall that, by definition, $v_{i}=x_{i}-x_{i-1}$. Thus, the result now follows.

Now we show that $f: \Gamma_{n} \mapsto \mathbb{N}^{n-1}$ such that $f(I)=\operatorname{pv}_{n}(I)$ is a one-to-one correspondence by constructing the inverse map.

Setup 2.9. Let $v=\left(v_{1}, v_{2}, \ldots, v_{n-1}\right) \in \mathbb{N}^{n-1}$. We will define two recursive sequences as follows:

(1) $t_{1}=0$ and $t_{i}=\left(v_{i}+t_{i-1}\right) \bmod i$, for $2 \leq i \leq n-1$.

(2) $l_{1}=v_{1}-1$ and $l_{i}=l_{i-1}+\frac{v_{i}+t_{i-1}-t_{i}}{i}$, for $2 \leq i \leq n-1$.

Notice that $0 \leq t_{i} \leq i-1$ for $1 \leq i \leq n-1$. Thus, $\left(t_{1}, t_{2}, \ldots, t_{n-1}\right)$ is the conversion vector of a permutation $\sigma=\left[\sigma_{1} \sigma_{2} \cdots \sigma_{n-1}\right]$ according to Proposition 2.6. Now let

$$
\mathcal{A}_{v}=\{0\} \cup\left\{n l_{i}+\sigma_{i}: 1 \leq i \leq n-1\right\} .
$$

Since $\sigma$ is a permutation on the set $\{1, \ldots, n-1\}, \mathcal{A}_{v}$ is a complete residue system modulo $n$ that contains 0 . Thus, $\mathcal{A}_{v}$ generates a numerical set $I\left(\mathcal{A}_{v}\right)=\left\{w+k n: w \in A_{v}, k \geq 0\right\}$ in $\Gamma_{n}$. We set $g: \mathbb{N}^{n-1} \mapsto \Gamma_{n}$ such that $g(v)=I\left(\mathcal{A}_{v}\right)$.

Theorem 2.10. The functions $f: \Gamma_{n} \mapsto \mathbb{N}^{n-1}$ given by $f(I)=p v_{n}(I)$ and $g: \mathbb{N}^{n-1} \mapsto$ $\Gamma_{n}$ by $g(v)=I\left(\mathcal{A}_{v}\right)$ are inverse functions. Therefore, the function $f$ is a one-to-one correspondence between numerical sets closed under addition by the element $n$ and $(n-1)$ tuples of positive integers.

Proof. Let $I \in \Gamma_{n}$ with $\operatorname{Ap}(I, n)=\left\{0, w_{1}, \ldots, w_{n-1}\right\}$, and write $w_{i}=n k_{i}+\pi_{i}$, where $0 \leq \pi_{i}<n$. Also let $v=\operatorname{pv}_{n}(I)$ and $\mathcal{A}_{v}=\{0\} \cup\left\{n l_{i}+\sigma_{i}: 0 \leq i \leq n-1\right\}$ as defined in Setup 2.9. It suffices to show that $\pi_{i}=\sigma_{i}$ and $k_{i}=l_{i}$ for all $1 \leq i \leq n-1$.

As noted before, $\left[\pi_{1} \pi_{2} \cdots \pi_{n-1}\right]$ is a permutation, and let $r=\left(r_{1}, r_{2}, \ldots, r_{n-1}\right)$ be its conversion vector. By Theorem 2.8, $r_{1}=0$, and $r_{i}=\left(v_{i}+r_{i-1}\right)-i\left(k_{i}-k_{i-1}\right)$. Since $0 \leq r_{i} \leq i-1$, it follows that $r_{i}=\left(v_{i}+r_{i-1}\right) \bmod i$, for $1 \leq i \leq n-1$. Referring to Setup 2.9. we find that $r_{i}=t_{i}$, for $1 \leq i \leq n-1$. Thus, $r$ is the conversion vector of both $\left[\pi_{1} \pi_{2} \cdots \pi_{n-1}\right]$ and $\left[\sigma_{1} \sigma_{2} \cdots \sigma_{n-1}\right]$, and we conclude the two permutations are equal.

Again, by Theorem 2.8 and Setup 2.9, $k_{1}=v_{1}-1=l_{1}$, and for $2 \leq i \leq n-1$,

$$
\begin{aligned}
k_{i}-k_{i-1} & =\frac{v_{i}+r_{i-1}-r_{i}}{i} \\
& =\frac{v_{i}+t_{i-1}-t_{i}}{i} \\
& =l_{i}-l_{i-1}
\end{aligned}
$$

We conclude that $k_{i}=l_{i}$, for $1 \leq i \leq n-1$. This shows that $g \circ f=i d_{\Gamma_{n}}$, and similarly, we obtain that $f \circ g=i d_{\mathbb{N}^{n-1}}$. Therefore, the function $f$ is a one-to-one correspondence. 
The next corollary is really a restatement of Theorem 2.10 ,

Corollary 2.11. Every numerical set closed under addition by the element $n \in \mathbb{N}$ has a unique position vector of length $n-1$. In particular, no two numerical semigroups have the same position vector. Moreover, every vector of length $n-1$ with entries in $\mathbb{N}$ is the position vector of a numerical set closed under addition by the element $n$.

The next example demonstrates what we have developed in this section.

Example 2.12. Let $S=\langle 6,16,20,21,29\rangle$. This is a numerical semigroup containing 6 , and hence $S \in \Gamma_{6}$. We can compute

$$
\operatorname{Ap}(S, 6)=\{0,16,20,21,29,37\}=\{0,6(2)+4,6(3)+2,6(3)+3,6(4)+5,6(6)+1\} .
$$

The permutation [42351] has conversion vector $(0,0,1,3,0)$. Thus,

$$
\begin{aligned}
& v_{1}=2+1=3 \\
& v_{2}=2(3-2)+(0-0)=2 \\
& v_{3}=3(3-3)+(1-0)=1 \\
& v_{4}=4(4-3)+(3-1)=6 \\
& v_{5}=5(6-4)+(0-3)=7 .
\end{aligned}
$$

So, we have $\operatorname{pv}_{6}(S)=(3,2,1,6,7)$.

Conversely, suppose we start with $v=(3,2,1,6,7) \in \mathbb{N}^{5}$. According to Setup 2.9 and Theorem 2.10, the $r_{i}$ 's are $(0,0,1,3,0)$ and the $k_{i}$ 's $(2,3,3,4,6)$. From the conversion vector $(0,0,1,3,0)$, we construct the corresponding permutation [42351] (see Example 2.7). Now,

$$
\begin{aligned}
& w_{0}=0 \\
& w_{1}=6(2)+4=16 \\
& w_{2}=6(3)+2=20 \\
& w_{3}=6(3)+3=21 \\
& w_{4}=6(4)+5=29 \\
& w_{5}=6(6)+1=37 .
\end{aligned}
$$

Thus, $S=\left\{w_{i}+c_{i} n: c_{i} \geq 0\right\}=\langle 6,16,20,21,29\rangle$.

\section{Position Vectors of NUMERICAL SEMigroups}

Now that we have a one-to-one correspondence between $\mathbb{N}^{n-1}$ and numerical sets closed under addition by $n$ established in the previous section, we want to know which position vectors correspond to semigroups. We provide a method for solving this problem and give an explicit answer for semigroups containing small numbers.

We begin with a necessary and sufficient condition for a complete residue system modulo $n$ that contains 0 to be the Apéry set of a numerical semigroup. This result is similar to others contained in [2, 4], and the proof is omitted. 
Lemma 3.1. Let $\mathcal{A}=\left\{w_{0}, w_{1}, \ldots, w_{n-1}\right\}$ be a complete residue system modulo $n$ that contains 0 , where $w_{0}<w_{1}<\cdots<w_{n-1}$. Then, $I(\mathcal{A})=\{w+k n: w \in \mathcal{A}, k \geq 0\}$ is a numerical semigroup if and only if $w_{i}+w_{j} \geq w_{l}$ whenever $w_{i}+w_{j} \equiv w_{l} \bmod n$ with $0<i \leq j<l$.

We can now translate Lemma 3.1 into a condition concerning the position vector, but first we need a few preliminary results.

Lemma 3.2. Let $I \in \Gamma_{n}$ be a numerical set with Apéry set $\operatorname{Ap}(I, n)=\left\{w_{0}, w_{1}, \ldots, w_{n-1}\right\}$, where $w_{0}<w_{i}<\cdots<w_{n-1}$. We set $w_{i}=n k_{i}+\pi_{i}$ for $0 \leq i \leq n-1$. If $v=\left(v_{1}, \ldots, v_{n-1}\right)$ is the position vector of $I$, then $k_{1}=v_{1}-1$ and

$$
k_{i}-k_{i-1}=\left\lfloor\frac{v_{i}-1}{i}\right\rfloor+\gamma_{i}
$$

where $\gamma_{1}=0$ and

$$
\gamma_{i}=\left\{\begin{array}{ll}
0 & \text { if } \pi_{i-1}<\pi_{i} \\
1 & \text { if } \pi_{i-1}>\pi_{i}
\end{array},\right.
$$

for $2 \leq i \leq n-1$.

Proof. It follows from Theorem 2.8 that $k_{1}=v_{1}-1$ and $v_{i}=i\left(k_{i}-k_{i-1}\right)+\left(r_{i}-r_{i-1}\right)$, for $2 \leq i \leq n-1$, where $r=\left(r_{1}, \ldots, r_{n-1}\right)$ is the conversion vector of $\pi=\left[\pi_{1} \cdots \pi_{n-1}\right]$. We rewrite this as $v_{i}-1=i\left(k_{i}-k_{i-1}-\gamma_{i}\right)+\left(i \gamma_{i}+r_{i}-r_{i-1}-1\right)$. If we show that $0 \leq i \gamma_{i}+r_{i}-r_{i-1}-1<i$ whenever $2 \leq i \leq n-1$, then it follows that

$$
k_{i}-k_{i-1}=\left\lfloor\frac{v_{i}-1}{i}\right\rfloor+\gamma_{i} .
$$

First suppose that $r_{i}>r_{i-1}$. By the definition of the conversion vector, we have $\pi_{i-1}<\pi_{i}$ and recall that $0 \leq r_{j} \leq j-1$ for all $1 \leq j \leq n-1$. Thus, $\gamma_{i}=0$ and $0 \leq r_{i}-r_{i-1}-1 \leq i-2$. Next, suppose that $r_{i} \leq r_{i-1}$ so that $\pi_{i-1}>\pi_{i}$. Then $\gamma_{i}=1$ and $1 \leq i+r_{i}-r_{i-1}-1 \leq i-1$, which completes the proof.

The next proposition will lead to a convenient equivalence relation on the elements of $\mathbb{N}^{n-1}$, i.e., the position vectors of numerical sets in $\Gamma_{n}$.

Proposition 3.3. Let $v=\left(v_{1}, \ldots, v_{m}\right)$ and $z=\left(z_{1}, \ldots, z_{m}\right)$ be two position vectors with associated conversion vectors and permutations denoted by $r$ and $\pi$ for $v$, and $s$ and $\sigma$ for z. Then the following are equivalent:

(1) $v_{i} \equiv z_{i} \bmod i$ for all $1 \leq i \leq m$

(2) $r=s$

(3) $\pi=\sigma$.

Proof. For (1) implies (2), by Setup 2.9, the conversion vector depends on the position vector modulo $i$ for the $i$-th entry. The equivalence of (2) and (3) follows from Proposition 2.6. Lastly, (2) implies (1) since, according to Theorem 2.8, $v_{i} \equiv r_{i}-r_{i-1} \bmod i$. 
Definition 3.4. We say two elements $v=\left(v_{1}, \ldots, v_{m}\right)$ and $z=\left(z_{1}, \ldots, z_{m}\right)$ of $\mathbb{N}^{m}$ are congruent, denoted by $v \sim z$, if $v_{i} \equiv z_{i} \bmod i$ for all $1 \leq i \leq m$. In this case, $v$ and $z$ have the same associated permutation $\pi$ and are said to be in the permutation class defined by $\pi$.

We can now present the main result of this section.

Theorem 3.5. Let $\left(v_{1}, v_{2}, \ldots, v_{m}\right)$ be a vector of positive integers in the permutation class defined by $\left[\pi_{1} \pi_{2} \cdots \pi_{m}\right]$. Also set $\gamma_{i}$ as in Lemma 3.2 and

$$
u_{i}=\left\lfloor\frac{v_{i}-1}{i}\right\rfloor .
$$

Then $\left(v_{1}, v_{2}, \ldots, v_{m}\right)$ is the position vector of a numerical semigroup if and only if

$$
\sum_{x=1}^{i}\left(u_{x}+\gamma_{x}\right)+\frac{\pi_{i}+\pi_{j}-\pi_{l}}{m+1} \geq \sum_{x=j+1}^{l}\left(u_{x}+\gamma_{x}\right),
$$

whenever $0<i \leq j<l$ and $\pi_{i}+\pi_{j} \equiv \pi_{l} \bmod (m+1)$.

Proof. Let $\left(v_{1}, v_{2}, \ldots, v_{m}\right)$ be the position vector of a numerical semigroup $S$ with Apéry set $\operatorname{Ap}(S, m+1)=\left\{w_{0}, \ldots, w_{m}\right\}$, where $w_{i}=(m+1) k_{i}+\pi_{i}$ for $1 \leq i \leq m$. If $0<i \leq j<l$ and $\pi_{i}+\pi_{j} \equiv \pi_{l} \bmod (m+1)$, then $w_{i}+w_{j} \equiv w_{l} \bmod (m+1)$ and by Lemma 3.1, we have $w_{i}+w_{j} \geq w_{l}$. Thus,

$$
\begin{aligned}
w_{i}+w_{j} & \geq w_{l} \\
k_{i}+\frac{\pi_{i}+\pi_{j}-\pi_{l}}{m+1} & \geq k_{l}-k_{j} \\
\sum_{x=1}^{i}\left(k_{x}-k_{x-1}\right)+\frac{\pi_{i}+\pi_{j}-\pi_{l}}{m+1} & \geq \sum_{x=j+1}^{l}\left(k_{x}-k_{x-1}\right) \\
\sum_{x=1}^{i}\left(u_{x}+\gamma_{x}\right)+\frac{\pi_{i}+\pi_{j}-\pi_{l}}{m+1} & \geq \sum_{x=j+1}^{l}\left(u_{x}+\gamma_{x}\right) .
\end{aligned}
$$

Essentially reversing these steps provides the converse argument and finishes the proof.

The next example applies Theorem 3.5 to numerical semigroups containing 3 .

Example 3.6. Every numerical set closed under addition by 3 belongs to one of two permutation classes, namely one defined by permutation [12] or the permutation [21]. We consider these two cases separately:

(1) For the permutation [12], we have $\pi_{1}+\pi_{1} \equiv \pi_{2} \bmod 3, \gamma_{1}=0$, and $\gamma_{2}=0$. Thus,

$$
\begin{aligned}
u_{1}+\frac{1+1-2}{3} & \geq u_{2} \\
u_{1} & \geq u_{2} .
\end{aligned}
$$


(2) For the permutation [21], we have $\pi_{1}+\pi_{1} \equiv \pi_{2} \bmod 3, \gamma_{1}=0$, and $\gamma_{2}=1$. Thus,

$$
\begin{aligned}
u_{1}+\frac{2+2-1}{3} & \geq u_{2}+1 \\
u_{1} & \geq u_{2} .
\end{aligned}
$$

We conclude that the vector $\left(v_{1}, v_{2}\right)$ corresponds to a numerical semigroup if and only if $u_{1} \geq u_{2}$, or equivalently,

$$
v_{1}-1 \geq\left\lfloor\frac{v_{2}-1}{2}\right\rfloor .
$$

Using the method derived from Theorem 3.5 and demonstrated in Example 3.6, we summarize the computational results for semigroups containing $n$ where $2 \leq n \leq 5$. We omit the details.

Theorem 3.7. Let $\left(v_{1}, v_{2}, \ldots, v_{n-1}\right)$ be an $(n-1)$-tuple of positive integers and set

$$
u_{i}=\left\lfloor\frac{v_{i}-1}{i}\right\rfloor \text {. }
$$

The following is a list necessary and sufficient conditions for $v$ to be the position vector of the a numerical semigroup containing $n$, for $2 \leq n \leq 5$.

- $n=2:\left(v_{1}\right)$ with no restriction

- $n=3:\left(v_{1}, v_{2}\right)$ such that $u_{1} \geq u_{2}$.

- $n=4:\left(v_{1}, v_{2}, v_{3}\right)$ with restrictions given in Table 1

\begin{tabular}{|c|c|}
\hline$\sim$ to one of & satisfying \\
\hline$(1,1,1),(1,2,3)$ & $u_{1} \geq u_{2}$ and $u_{1} \geq u_{3}$ \\
\hline$(1,1,2),(1,2,2)$ & $u_{1} \geq u_{3}$ \\
\hline$(1,2,1)$ & $u_{1} \geq u_{2}+u_{3}$ \\
\hline$(1,1,3)$ & $u_{1} \geq u_{2}+u_{3}+1$ \\
\hline
\end{tabular}

TABLE 1. Restrictions for a 3 -tuple to represent a semigroup

- $n=5:\left(v_{1}, v_{2}, v_{3}, v_{4}\right)$ with restrictions given in Table 2

In Theorem 3.7, $n$ is an element of the semigroup. By adding an extra restriction, we can force $n$ to be the multiplicity.

Proposition 3.8. Let $S$ be a semigroup containing $n$ with position vector $\left(v_{1}, v_{2}, \ldots, v_{n-1}\right)$. Then $n$ is the multiplicity of $S$ if and only if $v_{1}>1$.

Proof. We always have $v_{1} \geq 1$. If $v_{1}>1$, then the first nonzero element of $S$ is not in $\operatorname{Ap}(S, n)$. Thus the first nonzero element of $S$ cannot be smaller than $n$, and so $n$ is the multiplicity of $S$. If $v_{1}=1$, then the first nonzero element of $S$ is in $\operatorname{Ap}(S, n)$. Thus the first nonzero element of $S$ is smaller than $n$, and $n$ is not the multiplicity of $S$.

Remark 3.9. It follows that in Theorem 3.7, we can add the restriction $u_{1}>0$ to ensure that $n$ is the multiplicity of $S$. 


\begin{tabular}{|c|c|}
\hline$\sim$ to one of & satisfying \\
\hline$(1,1,1,1),(1,1,2,2)$, & $u_{1} \geq u_{2}, u_{1} \geq u_{3}, u_{1} \geq u_{4}$, \\
$(1,2,2,3),(1,2,3,4)$ & and $u_{1}+u_{2} \geq u_{3}+u_{4}$ \\
\hline$(1,2,1,2),(1,2,3,1)$ & $u_{1} \geq u_{2}$ and $u_{1} \geq u_{3}+u_{4}$ \\
\hline$(1,1,3,3),(1,1,1,4)$ & $u_{1} \geq u_{2}$ and $u_{1} \geq u_{3}+u_{4}+1$ \\
\hline$(1,1,1,2),(1,2,1,4)$ & $u_{1} \geq u_{2}+u_{3}, u_{1} \geq u_{4}$, \\
& and $u_{1}+u_{2} \geq u_{3}+u_{4}$ \\
\hline$(1,2,3,3),(1,1,3,1)$ & $u_{1} \geq u_{2}+u_{3}+1, u_{1} \geq u_{4}$, \\
& and $u_{1}+u_{2} \geq u_{3}+u_{4}$ \\
\hline$(1,1,1,3),(1,2,3,2)$, & $u_{1} \geq u_{2}+u_{3}+u_{4}+1$ \\
$(1,2,2,1),(1,1,2,4)$, & \\
$(1,1,2,3),(1,2,2,2)$ & $u_{1} \geq u_{2}+u_{3}+u_{4}+2$ \\
\hline$(1,1,3,4)$ & $u_{1} \geq u_{2}+u_{3}+u_{4}$ \\
\hline$(1,2,1,1)$ & $u_{1} \geq u_{2}+u_{3}$ and $u_{1} \geq u_{3}+u_{4}$ \\
\hline$(1,1,2,1),(1,2,1,3)$ & $u_{1} \geq u_{2}+u_{3}+1$ and $u_{1} \geq u_{3}+u_{4}+1$ \\
\hline$(1,2,2,4),(1,1,3,2)$ &
\end{tabular}

TABLE 2. Restrictions for a 4 -tuple to represent a semigroup

\section{REFERENCES}

[1] Barucci, V., Dobbs, D., Fontana, M.: Maximality properties in numerical semigroups and applications to one-dimensional analytically irreducible local domains. Mem. Amer. Math. Soc. 125, vii-77 (1997).

[2] Branco, M., García-García, J., García-Sanchez, P. A., Rosales, J.: Systems of inequalities and numerical semigroups. J. London Math. Soc. (2) 65, 611-623 (2002).

[3] García-Sánchez, P. A., Rosales, J. C.: Numerical semigroups. Developments in Mathematics, 20. Springer, New York (2009). ISBN 978-1-4419-0159-0.

[4] Kaplan, N.: Counting numerical semigroups by genus and some cases of a question of Wilf. J. Pure Appl. Algebra, (5) 216, 1016-1032 (2009).

[5] Marzuola, J., Miller, A.: Counting numerical sets with no small atoms. J. Combin. Theory Ser. A 117 (2010), no. 6, 650-667.

[6] Pellikaan, R., Torres, F.: On Weierstrass semigroups and the redundancy of improved geometric Goppa codes. IEEE Trans. Inform. Theory 45(7) (1999), 2512-2519.

[7] Pemmaraju, S. V., Skiena, S.: "Permutations and combinations" in computational discrete mathematics: combinatorics and graph theory with Mathematica. Cambridge University Press (2003). ISBN 978-0-5211-2146-0.

[8] Thompkins, C.: Machine attacks on problems whose variable are permutations. Proc. Symposia Applied Mathematics. McGraw-Hill, New York (1956). 